\title{
Adaptive Fuzzy Integral Terminal Sliding Mode Control of a Nonholonomic Wheeled Mobile Robot
}

\author{
Shuying Peng ${ }^{1,2}$ and Wuxi Shi ${ }^{2,3}$ \\ ${ }^{1}$ School of Mechanical Engineering, Tianjin Polytechnic University, Tianjin 300387, China \\ ${ }^{2}$ Tianjin Key Laboratory of Advanced Technology of Electrical Engineering and Energy, Tianjin 300387, China \\ ${ }^{3}$ School of Electrical Engineering and Automation, Tianjin Polytechnic University, Tianjin 300387, China \\ Correspondence should be addressed to Shuying Peng; psy19982002@163.com
}

Received 5 December 2016; Revised 13 March 2017; Accepted 16 March 2017; Published 5 April 2017

Academic Editor: Ashitava Ghosal

Copyright (c) 2017 Shuying Peng and Wuxi Shi. This is an open access article distributed under the Creative Commons Attribution License, which permits unrestricted use, distribution, and reproduction in any medium, provided the original work is properly cited.

\begin{abstract}
In this paper, the trajectory tracking problem is investigated for a nonholonomic wheeled mobile robot with parameter uncertainties and external disturbances. In this strategy, combining the kinematic model with the dynamic model, the actuator voltage is employed as the control input, and the uncertainties are approximated by a fuzzy logic system. An auxiliary velocity controller is integrated with an adaptive fuzzy integral terminal sliding mode controller, and a robust controller is employed to compensate for the lumped errors. It is proved that all the signals in the closed system are bounded and the auxiliary velocity tracking errors can converge to a small neighborhood of the origin in finite time. As a result, the tracking position errors converge asymptotically to zeros with faster response than other existing controllers. Simulation results demonstrate the effectiveness of the proposed strategy.
\end{abstract}

\section{Introduction}

A wheeled mobile robot (WMR) is an uncertain nonlinear MIMO dynamic system. When the WMR constrains the wheel's "pure rolling without slipping," it is also a typical kind of nonholonomic systems characterized by kinematic constraints. Such constraints are not integrable and can not be eliminated from the model equations. Given so many characteristics that are hard to handle, there has been tremendous research on the nonholonomic WMR (NWMR) in the past few decades.

The trajectory tracking problem is one of the most popular problems on the WMR. With an assumption of "perfect velocity tracking," the initial kinematic controller for the NWMR was designed in $[1,2]$. However, such an assumption is difficult to hold in practice for the dynamic model of the NWMR is neglected. Considering the kinematic model and the dynamic model of the NWMR together, based on backstepping technique, Fierro and Lewis [3] presented a dynamical extension that combines a kinematic controller with a torque controller. In this method, it is assumed that the dynamic structure of the NWMR and the parameters are completely known. However, in practical WMRs, there exist parameter uncertainties and external disturbances. In addition, wheel skidding and slipping may happen. To overcome these difficulties, the torus shaped rear wheels were used for three WMRs in $[4,5]$. The modeling and analysis were investigated to design the controller for the WMR in [6]. Meanwhile, a variety of nonlinear control techniques have been used by many researchers, such as adaptive control [7-11], robust adaptive control [12-14], adaptive fuzzy logic control [15-18], adaptive neural network control [19, 20], and sliding mode control [21-23], and several kinds of the aforementioned methodologies are integrated to solve this problem $[24,25]$.

One idea of some proposed literatures related to the trajectory tracking problem of the NWMR is that an auxiliary velocity controller is designed for the kinematic model of the NWMR to make the tracking position errors converge asymptotically to zeros, and a dynamic controller is designed for the dynamic model of the NWMR to make the auxiliary velocity tracking errors as small as possible. Meanwhile, a robust controller is employed to compensate the total uncertainties. For instance, by virtue of the universal 
approximation property of the fuzzy logic system (FLS) [2631], a control structure combining a kinematic controller with a dynamic controller plus a fuzzy compensator was proposed in [15]. A complete control law based on a kinematic controller and an adaptive fuzzy sliding mode controller was developed for a NWMR in the presence of dynamic uncertainties as well in [25]. These dynamic controllers share a common idea of choosing the wheel torque as the control input. However, as stated in [17], the wheel is driven by the actuator in reality. Hence, the resulting electrically driven mobile robot (the robot kinematics, robot dynamics, and wheel actuator dynamics) is represented as a third-order system. So most of existing torque controllers designed with respect to the second-order, that is, the wheel actuator dynamics, have been neglected and might degrade the performance of the tracking control. Therefore, it is more reasonable to use the actuator voltage as the control input. For realizing the trajectory tracking of the NWMR with high performance, the wheel actuator dynamics are combining with the dynamics of the NWMR and the actuator voltage is employed as the control input in $[8,16-18]$. All these dynamic controllers can guarantee that the auxiliary velocity tracking errors converge to an adjustable neighborhood of the origin as time goes to infinity. However, the finite time convergence of the auxiliary velocity tracking errors can not be guaranteed.

The terminal sliding mode control (TSMC), which was first proposed in $[32,33]$, is an effective scheme to guarantee the finite time convergence of the auxiliary velocity tracking errors. However, the initial TSMC may cause the singularity problem around the equilibrium [34], which would result in an unbounded control signal. In order to avoid this problem, a nonsingular terminal sliding mode control (NTSMC) was developed in [35-38]. The continuous nonsingular terminal sliding mode [36] has been extended into a class of MIMO nonlinear systems [39]. Furthermore, using integral operation, an integral terminal sliding mode control (ITSMC) was presented in $[40,41]$ for a class of first-order systems. Apart from finite time convergence and nonsingularity, in the ITSMC design, the system can also start on the integral terminal sliding mode surface from the initial time instant. Therefore, the reaching time to the sliding mode surface is eliminated.

Based on the previous results, this paper addresses the trajectory tracking problem for the NWMR with parameter uncertainties and external disturbances. Combining the kinematic model with the dynamic model, a control strategy is proposed which integrates an auxiliary velocity controller with an adaptive fuzzy integral terminal sliding mode controller. In this control strategy, using the universal approximation property of the FLS, the uncertainties are approximated by a fuzzy logic system and a robust controller is employed to compensate for the lumped errors. Meanwhile, instead of the wheel torque, the actuator voltage is employed as the control input. The main originality of the proposed control strategy is that the adaptive fuzzy integral terminal sliding mode controller can guarantee the finite time convergence of the auxiliary velocity tracking errors. It is proved that all the signals in the closed system are bounded and the auxiliary velocity tracking errors converge to a small

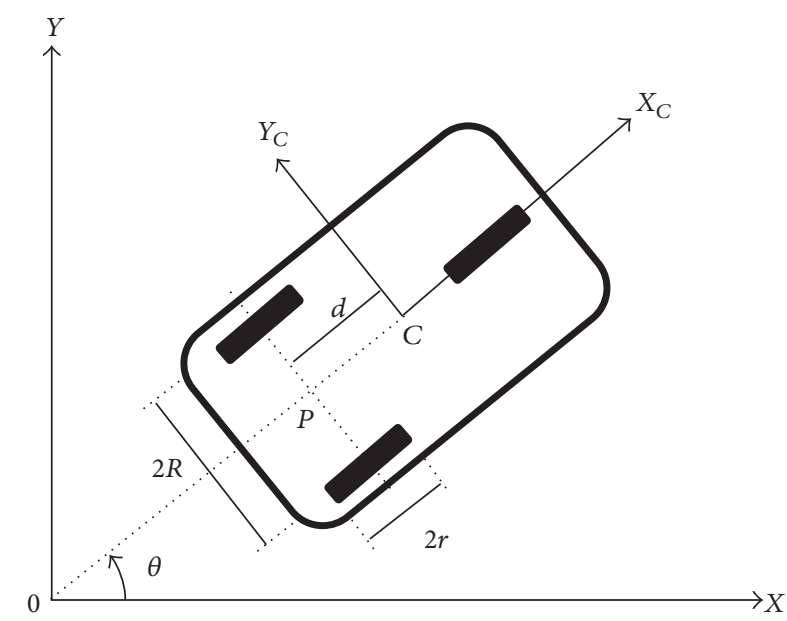

Figure 1: A wheeled mobile robot.

neighborhood of the origin in finite time. Therefore, the tracking position errors converge asymptotically to zeros with faster response than other existing controllers. Simulation results demonstrate the effectiveness of the proposed strategy.

The remainder of this paper is organized as follows. Section 2 reviews some basics of the model of the NWMR, the ITSMC, and the FLS. By use of the ITSMC and the FLS, a control strategy is proposed which integrates an auxiliary velocity controller with an adaptive fuzzy integral terminal sliding mode controller in Section 3. Section 4 gives simulation results to illustrate our results. Conclusions are given in Section 5.

\section{Preliminaries}

In this section, we will review some basics of the model of the NWMR, the ITSMC, and the FLS briefly.

2.1. Model of the Nonholonomic Wheeled Mobile Robot. We consider a typical example of the WMR, which is called Type $(2,0)$ WMR in [6]. Such a WMR is composed of two deriving wheels and one passive wheel. The two deriving wheels are controlled independently by two actuators to achieve the motion and orientation, and the passive wheel prevents the robot from tipping over as it moves on a plane. Figure 1 describes the posture of the WMR in Cartesian coordinates. Both driving wheels with the same radius $r$ are mounted on the same axis and separated by $2 R$. The center of mass of the WMR is located at $C$, and $P$ is located in the midpoint of the two driving wheels of the WMR. The distance between $P$ and $C$ is $d$. When the electrical part of the actuator is taken into account, the kinematic equation and the dynamic equation of the NWMR can be written as follows from $[16,19]$ :

$$
\begin{aligned}
& \dot{q}=s(q) \vartheta, \\
& \bar{M}(q) \dot{\vartheta}+\bar{V}(q, \dot{q}) \vartheta+\bar{F}(\vartheta)+\bar{\tau}_{d} \\
& \quad=\frac{N K_{T}}{R_{a}} \bar{B} u-\frac{N^{2} K_{T} K_{b}}{R_{a}} \bar{B} X \vartheta,
\end{aligned}
$$


where

$$
\begin{aligned}
S(q) & =\left[\begin{array}{cc}
\cos \theta & -d \sin \theta \\
\sin \theta & d \cos \theta \\
0 & 1
\end{array}\right], \\
\bar{M}(q) & =\left[\begin{array}{cc}
m & 0 \\
0 & I-m d^{2}
\end{array}\right], \\
\bar{V}(q, \dot{q}) & =\left[\begin{array}{ll}
0 & 0 \\
0 & 0
\end{array}\right], \\
\bar{B} & =\frac{1}{r}\left[\begin{array}{cc}
1 & 1 \\
R & -R
\end{array}\right], \\
X & =\bar{B}^{T} .
\end{aligned}
$$

$q=[x, y, \theta]^{T}, C(x, y)$ is the coordinate of $C$ in the global coordinate frame $X O Y$, and $\theta$ is the orientation of the local coordinate frame $X_{C} C Y_{C}$ attached on the WMR platform measured from $X$ axis and is also called the heading angle of the WMR. $\vartheta=[\nu, \omega]^{T}$, where $\nu$ and $\omega$ are the linear velocity of the point $P$ along the robot axis and angle velocity, respectively. $\bar{M}(q)$ is the inertia matrix, $\bar{V}(q, \dot{q})$ is the centripetal and Coriolis matrix, $\bar{F}(9) \in R^{2 \times 1}$ is the surface friction, and $\bar{\tau}_{d} \in R^{2 \times 1}$ denotes bounded unknown disturbances including unstructured unmodeled dynamics. $N$ is the gear ration, $K_{T}$ is the motor torque constant, $K_{b}$ is the counter electromotive force coefficient, and $R_{a}$ is the electric resistance. $u=\left[u_{1}, u_{2}\right]^{T}$ is the actuator voltage input vector.

Several properties of the NWMR are given as follows [19].

Property 1 . The matrix $\bar{M}(q)$ is symmetric and positive definite.

Property 2. The matrix $\bar{M}(q)$ is bounded; that is, there exist positive constants $m_{1}$ and $m_{2}$ satisfying $m_{1}\|x\|^{2} \leq$ $x^{T} \bar{M}(q) x \leq m_{2}\|x\|^{2}$, for all $x \in R^{2}$.

Property 3. The matrix $\bar{M}(q)-2 \bar{V}(q, \dot{q})$ is skew symmetric resulting in the following characteristic: $x^{T}(\bar{M}(q)-$ $2 \bar{V}(q, \dot{q})) x=0$ for all $x \in R^{2}$.

In view of the dynamic model of the NWMR, (2) is a firstorder system; the ITSM can be utilized so that the finite time convergence of the auxiliary velocity tracking errors of the NWMR is obtained.

2.2. Integral Terminal Sliding Mode. Now, a new form of the integral terminal sliding mode is defined as

$$
s=x(t)-x(0)+\beta \int_{0}^{t}|x(\tau)|^{\gamma} \operatorname{sign}(x(\tau)) d \tau,
$$

where $x \in R$ is the system state variable, $\beta>0$, and $0<\gamma<1$, which generalizes the integral terminal sliding mode [41]

$$
s=x(t)+\beta \int_{0}^{t} x^{q / p}(\tau) d \tau,
$$

where $\beta>0$ and $p$ and $q$ are odd integers satisfying $p>q>$ 0.

Remark 1. It is worthwhile to notice that the range of the power $\gamma$ is larger than that of the power $q / p$. Meanwhile, by means of the basic theorem of differential and integral calculus [42], the integral terminal sliding mode (4) is continuous and differentiable although the absolute and signum operators are involved. Besides these properties, from (4), it is obvious that $s(0)=0$ without the prior knowledge of the parameter $\beta$. This implies that the system starts on the integral terminal sliding mode surface (4) from the initial time instant much easily.

Furthermore, on the sliding surface, $s=0$, which results in

$$
\dot{x}(t)+\beta|x(t)|^{\gamma} \operatorname{sign}(x(t))=0 .
$$

The finite time $t_{s}$ that is taken from $x(0) \neq 0$ to $x\left(t_{s}\right)=0$ is given by

$$
t_{s}=\frac{1}{\beta(1-\gamma)}|x(0)|^{1-\gamma} .
$$

As we stated previously, there exist parameter uncertainties and unknown disturbances in practical WMR. Taking these factors into account, an unknown nonlinear function is contained in the model of the NWMR. We will use the FLS to approximate this function.

2.3. Fuzzy Logic Systems. In this section, the FLS is discussed briefly. The basic configuration of an FLS consists of four components: fuzzifier, fuzzy rule base, fuzzy inference engine, and defuzzifier. The fuzzy rule base is a collection of IF-THEN rules and the $l$ th fuzzy rule is written as

$$
R^{l}: \text { IF } x_{1} \text { is } F_{1}^{l} \text { and... and } x_{n} \text { is } F_{n}^{l} \text {, THEN } y \text { is } G^{l},
$$

where $F_{i}^{l}$ and $G^{l}$ are fuzzy sets, associating with fuzzy membership functions $\mu_{F_{i}^{l}}\left(x_{i}\right)$ and $\mu_{G^{l}}(y)$, respectively, $i=$ $1, \ldots, n, l=1, \ldots, m, m$ is the number of rules.

Based on these fuzzy IF-THEN rules, the FLS performs a mapping from an input vector $x=\left[x_{1}, \ldots, x_{n}\right]^{T} \in R^{n}$ to an output variable $y \in R$. If we use the strategy of singleton fuzzifier, product inference, and center-average defuzzifier, the output of the FLS can be defined as follows:

$$
y(x)=\frac{\sum_{l=1}^{m} y^{l}\left(\prod_{i=1}^{n} \mu_{F_{i}^{l}}\left(x_{i}\right)\right)}{\sum_{l=1}^{m} \prod_{i=1}^{n} \mu_{F_{i}^{l}}\left(x_{i}\right)},
$$

where $y^{l}$ is the point in $G^{l}$ at which $\mu_{G^{l}}(y)$ obtains its maximum value 1 .

For simplicity, $y(x)$ can be written in the following compact form:

$$
y(x)=\widehat{\theta}^{T} \xi(x):=\widehat{f}(x \mid \widehat{\theta}),
$$

where $\widehat{\theta}=\left[y^{1}, \ldots, y^{m}\right]^{T}$ is called the unknown parameter vector which is to be updated and $\xi(x)=\left[\xi^{1}(x), \ldots\right.$, $\left.\xi^{m}(x)\right]^{T}$ is called the fuzzy basis function vector, $\xi^{l}(x)=$ $\prod_{i=1}^{n} \mu_{F_{i}^{l}}\left(x_{i}\right) / \sum_{l=1}^{m} \prod_{i=1}^{n} \mu_{F_{i}^{l}}\left(x_{i}\right), l=1,2, \ldots, m$. 
Lemma 2 (see [31]). Let $f(x)$ be a continuous function defined on a compact set $\Omega$. Then, for any constant $\varepsilon>0$, there exists $a$ fuzzy system (9) such that $\sup _{x \in \Omega}|f(x)-\widehat{f}(x \mid \widehat{\theta})| \leq \varepsilon$.

\section{Controller Design}

It is easy to see that the posture of the NWMR $q(t)=[x(t)$, $y(t), \theta(t)]^{T}$ satisfies the following equations from (1):

$$
\begin{aligned}
& \dot{x}=v \cos \theta-\omega d \sin \theta, \\
& \dot{y}=v \sin \theta+\omega d \cos \theta, \\
& \dot{\theta}=\omega .
\end{aligned}
$$

It is assumed that the reference trajectory $q_{r}(t)=\left[x_{r}(t)\right.$, $\left.y_{r}(t), \theta_{r}(t)\right]^{T}$ is generated by a reference NWMR with the kinematic equation as (10):

$$
\begin{aligned}
& \dot{x}_{r}=v_{r} \cos \theta_{r}-\omega_{r} d \sin \theta_{r}, \\
& \dot{y}_{r}=v_{r} \sin \theta_{r}+\omega_{r} d \cos \theta_{r}, \\
& \dot{\theta}_{r}=\omega_{r} .
\end{aligned}
$$

The objective of the trajectory tracking control is to design a strategy such that $q(t)$ converges asymptotically to $q_{r}(t)$, while all signals in the derived closed-loop system remain bounded. In this study, an auxiliary velocity controller $\vartheta_{c}$ is designed for the kinematic model (1) to meet the control objective. Then, the actuator voltage control input $u$ is designed for the dynamic model (2) such that $\vartheta$ converges to $\vartheta_{c}$ which is designed at the first step in finite time.

Remark 3. As pointed out in [18], the classical auxiliary velocity controller [1] adopted in [3, 16, 18, 19, 25] can only guarantee that $q(t)$ converges asymptotically to $q_{r}(t)$ when $d$ equals zero. However, $d$ does not equal zero in general. Therefore, the reference point of the practical NWMR is not in accordance with the desired point of the reference NWMR, which results in incomplete tracking of the posture. In this paper, we modify the kinematic model of the reference NWMR as (11) and adopt another auxiliary velocity controller [43].

3.1. An Auxiliary Velocity Controller Design. We define the tracking position errors as the difference between the center of mass $C$ of the NWMR and the desired point of the reference NWMR as follows $[16,19]$ :

$$
e_{p}=\left[\begin{array}{l}
e_{1} \\
e_{2} \\
e_{3}
\end{array}\right]=\left[\begin{array}{ccc}
\cos \theta & \sin \theta & 0 \\
-\sin \theta & \cos \theta & 0 \\
0 & 0 & 1
\end{array}\right]\left[\begin{array}{l}
x_{r}-x \\
y_{r}-y \\
\theta_{r}-\theta
\end{array}\right] .
$$

The first derivative of the error yields

$$
\begin{aligned}
{\left[\begin{array}{l}
\dot{e}_{1} \\
\dot{e}_{2} \\
\dot{e}_{3}
\end{array}\right]=} & {\left[\begin{array}{cc}
-1 & e_{2} \\
0 & -e_{1}-d \\
0 & -1
\end{array}\right]\left[\begin{array}{l}
v \\
\omega
\end{array}\right] } \\
& +\left[\begin{array}{c}
v_{r} \cos e_{3}-\omega_{r} d \sin e_{3} \\
v_{r} \sin e_{3}+\omega_{r} d \cos e_{3} \\
\omega_{r}
\end{array}\right] .
\end{aligned}
$$

Therefore, the objective of this study becomes the design of an auxiliary velocity controller to make the tracking position errors asymptotically converge to zeros. In this study, according to [43], the auxiliary velocity controller is designed as

$$
\vartheta_{c}=\left[\begin{array}{c}
\nu_{c} \\
\omega_{c}
\end{array}\right]=\left[\begin{array}{c}
v_{r} \cos e_{3}+k_{1}\left(e_{1}+d-d \cos e_{3}\right) \\
\omega_{r}+k_{3} \nu_{r}\left(e_{2}-d \sin e_{3}\right)+k_{2} \sin e_{3}
\end{array}\right],
$$

where $k_{1}, k_{2}, k_{3}>0$ are design parameters.

Substituting (14) into (13), the closed-loop kinematic equation can be written as

$$
\begin{aligned}
& {\left[\begin{array}{c}
\dot{e}_{1} \\
\dot{e}_{2} \\
\dot{e}_{3}
\end{array}\right]} \\
& =\left[\begin{array}{cc}
-1 & e_{2} \\
0 & -e_{1}-d \\
0 & -1
\end{array}\right]\left[\begin{array}{c}
v_{r} \cos e_{3}+k_{1}\left(e_{1}+d-d \cos e_{3}\right) \\
\omega_{r}+k_{3} v_{r}\left(e_{2}-d \sin e_{3}\right)+k_{2} \sin e_{3}
\end{array}\right] \\
& +\left[\begin{array}{c}
v_{r} \cos e_{3}-\omega_{r} d \sin e_{3} \\
v_{r} \sin e_{3}+\omega_{r} d \cos e_{3} \\
\omega_{r}
\end{array}\right] .
\end{aligned}
$$

Assumption 4 (see [8]). The reference velocities $\vartheta_{r}=\left[\nu_{r}, \omega_{r}\right]^{T}$ and $\dot{\nu}_{r}$ are bounded.

Lemma 5. For the kinematic model (1) of the NWMR satisfying Assumption 4, the auxiliary velocity controller (14) will ensure that the tracking position errors converge asymptotically to zeros.

Proof. Consider the following Lyapunov function candidate

$$
\begin{aligned}
V_{1}= & \frac{1}{2}\left(e_{1}+d-d \cos e_{3}\right)^{2}+\frac{1}{2}\left(e_{2}-d \sin e_{3}\right)^{2} \\
& +\frac{1-\cos e_{3}}{k_{3}} .
\end{aligned}
$$

Differentiating $V_{1}$ with respect to time, we have

$$
\begin{aligned}
\dot{V}_{1}= & \left(e_{1}+d-d \cos e_{3}\right)\left(\dot{e}_{1}+d \dot{e}_{3} \sin e_{3}\right) \\
& +\left(e_{2}-d \sin e_{3}\right)\left(\dot{e}_{2}-d \dot{e}_{3} \cos e_{3}\right)+\frac{1}{k_{3}} \dot{e}_{3} \sin e_{3} .
\end{aligned}
$$


Replacing (15) into (17) and after some manipulations, one obtains

$$
\dot{V}_{1}=-k_{1}\left(e_{1}+d-d \cos e_{3}\right)^{2}-\frac{k_{2}}{k_{3}} \sin ^{2} e_{3} \leq 0 .
$$

Therefore, the tracking position error $e_{P}=\left[e_{1}, e_{2}, e_{3}\right]^{T}$ is bounded. With Assumption $4, \vartheta_{c}$ and $\dot{e}_{P}=\left[\dot{e}_{1}, \dot{e}_{2}, \dot{e}_{3}\right]^{T}$ are bounded. So $\ddot{V}_{1}$ is bounded and $\dot{V}_{1}$ is uniformly continuous accordingly. By Barbalat's lemma [44], $\dot{V}_{1} \rightarrow 0$ as $t \rightarrow \infty$, which implies that $e_{1} \rightarrow 0$ and $e_{3} \rightarrow 0$ as $t \rightarrow \infty$.

From (15), one obtains

$$
\dot{e}_{3}=-k_{3} v_{r}\left(e_{2}-d \sin e_{3}\right)-k_{2} \sin e_{3} .
$$

Using Barbalat's lemma again, $\dot{e}_{3} \rightarrow 0$ as $t \rightarrow \infty$, which implies that $e_{2} \rightarrow 0$ as $t \rightarrow \infty$.

Hence, $e_{P} \rightarrow 0$ as $t \rightarrow \infty$; that is, the tracking position errors converge asymptotically to zeros.

Now, it remains to design the actuator voltage control input so that the desired velocities $\vartheta_{c}$ can be obtained in finite time.

3.2. Adaptive Integral Terminal Sliding Mode Controller Design. In this study, the auxiliary velocity tracking error is defined as

$$
e_{\vartheta}=\left[e_{\vartheta 1}, e_{\vartheta 2}\right]^{T}=\vartheta_{c}-\vartheta
$$

Consequently, the dynamic equation (2) of the NWMR can be rewritten as

$$
\begin{aligned}
\bar{M}(q) \dot{e}_{\vartheta}= & -\bar{V}(q, \dot{q}) e_{\vartheta}+\frac{N^{2} K_{T} K_{b}}{R_{a}} \bar{B} X \vartheta+\bar{M}(q) \dot{\vartheta}_{c} \\
& +\bar{V}(q, \dot{q}) \vartheta_{c}+\bar{F}(\vartheta)+\bar{\tau}_{d}-\frac{N K_{T}}{R_{a}} \bar{B} u .
\end{aligned}
$$

A continuous nonsingular integral terminal sliding mode is defined as in the form (4):

$$
\begin{aligned}
S & =\left[\begin{array}{l}
s_{1} \\
s_{2}
\end{array}\right] \\
& =\left[\begin{array}{l}
e_{\vartheta 1}-e_{91}(0)+\beta_{1} \int_{0}^{t}\left|e_{91}\right|^{\gamma_{1}} \operatorname{sign}\left(e_{\vartheta 1}\right) d \tau \\
e_{92}-e_{92}(0)+\beta_{2} \int_{0}^{t}\left|e_{92}\right|^{\gamma_{2}} \operatorname{sign}\left(e_{92}\right) d \tau
\end{array}\right],
\end{aligned}
$$

where $\beta_{i}>0,0<\gamma_{i}<1, i=1,2$.

Denote

$$
\begin{aligned}
\Lambda & =\operatorname{diag}\left(\beta_{1}, \beta_{2}\right), \\
\operatorname{sig}\left(e_{\vartheta}\right)^{\gamma} & =\left[\left|e_{\vartheta 1}\right|^{\gamma_{1}} \operatorname{sign}\left(e_{\vartheta 1}\right),\left|e_{\vartheta 2}\right|^{\gamma_{2}} \operatorname{sign}\left(e_{\vartheta 2}\right)\right]^{T} .
\end{aligned}
$$

$S$ can be rewritten as follows:

$$
S=e_{\vartheta}-e_{\vartheta}(0)+\Lambda \int_{0}^{t} \operatorname{sig}\left(e_{\vartheta}\right)^{\gamma} d \tau
$$

Utilizing $S$ and its derivative with respect to time, (21) can be arranged as follows:

$$
\begin{aligned}
\bar{M}(q) \dot{S}= & -\bar{V}(q, \dot{q}) S+\frac{N^{2} K_{T} K_{b}}{R_{a}} \bar{B} X \vartheta+f(x) \\
& -\frac{N K_{T}}{R_{\mathrm{a}}} \bar{B} u
\end{aligned}
$$

where

$$
\begin{aligned}
f(x)= & {\left[f_{1}(x), f_{2}(x)\right]^{T} } \\
= & \bar{M}(q) \dot{\vartheta}_{c}+\bar{M}(q) \Lambda \operatorname{sig}\left(e_{\vartheta}\right)^{\gamma}+\bar{V}(q, \dot{q}) \vartheta_{c} \\
& -\bar{V}(q, \dot{q}) e_{\vartheta}(0)+\bar{V}(q, \dot{q}) \Lambda \int_{0}^{t} \operatorname{sig}\left(e_{\vartheta}\right)^{\gamma} d \tau \\
& +\bar{F}(\vartheta)+\bar{\tau}_{d}
\end{aligned}
$$

and $x=\left[\vartheta_{c}^{T}, \dot{\vartheta}_{c}^{T}, \vartheta^{T}\right]^{T}$.

If $f(x)$ is known, let the actuator voltage control input

$$
\begin{aligned}
u= & \frac{R_{a}}{N K_{T}} \bar{B}^{-1}\left(\frac{N^{2} K_{T} K_{b}}{R_{a}} \bar{B} X \vartheta+f(x)+K_{1} S\right. \\
& \left.+K_{2} \operatorname{sig}(S)^{\rho}\right)
\end{aligned}
$$

where $K_{1}=\operatorname{diag}\left(k_{11}, k_{12}\right), K_{2}=\operatorname{diag}\left(k_{21}, k_{22}\right), k_{1 i}>0, k_{2 i}>$ $0, i=1,2$, and $0<\rho<1$.

Substituting (27) into (25), the closed-loop dynamic equation can be written as

$$
\bar{M}(q) \dot{S}=-\bar{V}(q, \dot{q}) S-K_{1} S-K_{2} \operatorname{sig}(S)^{\rho} .
$$

Multiplying $S^{T}$ by (28) yields

$$
S^{T} \bar{M}(q) \dot{S}=-S^{T} \bar{V}(q, \dot{q}) S-S^{T} K_{1} S-S^{T} K_{2} \operatorname{sig}(S)^{\rho} .
$$

Theorem 6. For the dynamic model (2) of the NWMR with a known function $f(x)$ (26), if the integral terminal sliding mode is chosen as (22) and the actuator voltage control input is designed as (27), then the integral terminal sliding mode $S$ and the auxiliary velocity tracking error $e_{9}$ will converge to zeros in finite time.

To prove Theorem 6, we introduce two lemmas.

Lemma 7 (see [36]). Suppose $a_{1}, a_{2}, \ldots, a_{n}$ and $0<p<2$ are all positive numbers, then the following inequality holds:

$$
\left(a_{1}^{2}+a_{2}^{2}+\cdots+a_{n}^{2}\right)^{p} \leq\left(a_{1}^{p}+a_{2}^{p}+\cdots+a_{n}^{p}\right)^{2} .
$$

Lemma 8 (see [36]). An extended Lyapunov description of finite time stability can be given with the form of fast terminal sliding mode as

$$
\dot{V}(x)+\alpha V(x)+\beta V^{\gamma}(x) \leq 0, \quad \alpha, \beta>0,0<\gamma<1,
$$

and the settling time can be given by

$$
t_{r} \leq \frac{1}{\alpha(1-\gamma)} \ln \frac{\alpha V^{1-\gamma}(x(0))+\beta}{\beta} .
$$


Proof of Theorem 6. Consider the following Lyapunov function candidate:

$$
V_{2}=\frac{1}{2} S^{T} \bar{M}(q) S
$$
yields

Differentiating $V_{2}$ with respect to time and using (29)

$$
\begin{aligned}
\dot{V}_{2}= & S^{T} \bar{M}(q) \dot{S}+\frac{1}{2} S^{T} \dot{\bar{M}}(q) S \\
= & \frac{1}{2} S^{T}(\dot{\bar{M}}(q)-2 \bar{V}(q, \dot{q})) S-S^{T} K_{1} S \\
& -S^{T} K_{2} \operatorname{sig}(S)^{\rho} .
\end{aligned}
$$

From Property 3, which makes the first term zero, $\dot{V}_{2}$ becomes

$$
\dot{V}_{2}=-S^{T} K_{1} S-S^{T} K_{2} \operatorname{sig}(S)^{\rho} .
$$

Denote $\lambda_{i}=\min \left\{k_{i 1}, k_{i 2}\right\}, i=1,2$; the following inequality holds:

$$
\dot{V}_{2} \leq-\lambda_{1} \sum_{i=1}^{2} s_{i}^{2}-\lambda_{2} \sum_{i=1}^{2}\left|s_{i}\right|^{\rho+1}
$$

Applying Lemma 7 into (36), one obtains

$$
\begin{aligned}
\dot{V}_{2} \leq & -\lambda_{1} \frac{2}{m_{2}} \sum_{i=1}^{2} \frac{m_{2}}{2} s_{i}^{2} \\
& -\lambda_{2}\left(\frac{2}{m_{2}}\right)^{(\rho+1) / 2}\left[\sum_{i=1}^{2} \frac{m_{2} s_{i}^{2}}{2}\right]^{(\rho+1) / 2} .
\end{aligned}
$$

Utilizing Property 2, we have

$$
\dot{V}_{2} \leq-\lambda_{1} \frac{2}{m_{2}} V_{2}-\lambda_{2}\left(\frac{2}{m_{2}}\right)^{(\rho+1) / 2} V_{2}^{(\rho+1) / 2},
$$

or

$$
\dot{V}_{2}+\lambda_{1} \frac{2}{m_{2}} V_{2}+\lambda_{2}\left(\frac{2}{m_{2}}\right)^{(\rho+1) / 2} V_{2}^{(\rho+1) / 2} \leq 0 .
$$

From Lemma 8, it follows that $S$ will converge to zero in finite time

$$
\begin{aligned}
t_{r} & \leq \frac{1}{\lambda_{1}\left(2 / m_{2}\right)(1-(\rho+1) / 2)} \\
& \cdot \ln \frac{\lambda_{1}\left(2 / m_{2}\right) V_{2}^{1-(\rho+1) / 2}(0)+\lambda_{2}\left(2 / m_{2}\right)^{(\rho+1) / 2}}{\lambda_{2}\left(2 / m_{2}\right)^{(\rho+1) / 2}} .
\end{aligned}
$$

Moreover, on the sliding mode surface, according to (7),

$$
t_{s i}=\frac{1}{\beta_{i}\left(1-\gamma_{i}\right)}\left|e_{9 i}(0)\right|^{1-\gamma_{i}}, \quad i=1,2 .
$$

Therefore, the auxiliary velocity tracking error $e_{9}$ will converge to zero in finite time $t=t_{r}+\max \left\{t_{s 1}, t_{s 2}\right\}$.
Due to the fact that $f(x)$ contains all the mobile robot parameters (such as mass, moment of inertia, and friction coefficients) and external disturbances, in the following, we assume that $f_{i}(x), i=1,2$, can be approximated by the following FLS:

$$
\widehat{f}_{i}\left(x \mid \widehat{\theta}_{f i}\right)=\widehat{\theta}_{f i}^{T} \xi_{f i}(x),
$$

where $\xi_{f i}(x)$ is the fuzzy basis function vector and $\widehat{\theta}_{f i}$ is the parameter vector of each fuzzy system designed later.

Define the optimal approximation parameters $\widehat{\theta}_{f i}^{*}$ as follows:

$$
\widehat{\theta}_{f i}^{*}=\underset{\widehat{\theta}_{f i} \in \Omega_{f i}}{\operatorname{argmin}}\left[\sup _{x \in U}\left|f_{i}(x)-\widehat{f}_{i}\left(x \mid \widehat{\theta}_{f i}\right)\right|\right],
$$

where $\Omega_{f i}$ is the compact set of allowable controller parameters. Moreover, the parameter error and the minimum approximation error are defined as $\widetilde{\theta}_{f i}=\widehat{\theta}_{f i}^{*}-\widehat{\theta}_{f i}$ and $\omega_{f i}(x)=$ $f_{i}(x)-\widehat{f}_{i}\left(x \mid \widehat{\theta}_{f i}^{*}\right)$, respectively.

Assumption 9. For $i=1,2, \omega_{f i}$ is bounded. That is, there exists an unknown constant $\bar{\omega}_{f i}$ such that $\left|\omega_{f i}(x)\right|<\bar{\omega}_{f i}$.

Denote

$$
\begin{aligned}
& \widehat{f}\left(x \mid \widehat{\theta}_{f}\right)=\left[\widehat{f}_{1}\left(x \mid \widehat{\theta}_{f 1}\right), \widehat{f}_{2}\left(x \mid \widehat{\theta}_{f 2}\right)\right]^{T}, \\
& \hat{f}\left(x \mid \theta_{f}^{*}\right)=\left[\widehat{f}_{1}\left(x \mid \theta_{f 1}^{*}\right), \widehat{f}_{2}\left(x \mid \theta_{f 2}^{*}\right)\right]^{T} .
\end{aligned}
$$

By using the fuzzy approximation $\widehat{f}\left(x \mid \hat{\theta}_{f}\right)$ instead of $f(x)$, the following control law from (27) is obtained:

$$
\begin{aligned}
u= & \frac{R_{a}}{N K_{T}} \bar{B}^{-1}\left(\frac{N^{2} K_{T} K_{b}}{R_{a}} \bar{B} X \vartheta+\widehat{f}\left(x \mid \hat{\theta}_{f}\right)+K_{1} s\right. \\
& \left.+K_{2} \operatorname{sig}(s)^{\rho}+u_{r}\right),
\end{aligned}
$$

where $u_{r}=\left[u_{r 1}, u_{r 2}\right]^{T}$ is a robust controller, which is designed as

$$
u_{r}= \begin{cases}\frac{\left(\widehat{\varepsilon}_{f}+\sigma\right) s}{\|s\|}, & s \neq 0, \\ 0, & s=0,\end{cases}
$$

$\widehat{\mathcal{\varepsilon}}_{f}$ is the estimate of $\sum_{i=1}^{2} \bar{\omega}_{f i}, \widetilde{\mathcal{\varepsilon}}_{f}=\widehat{\varepsilon}_{f}-\sum_{i=1}^{2} \bar{\omega}_{f i}$, and $\sigma$ is a positive constant.

Remark 10. It is noticed that the robust controller $u_{r}(46)$ is similar to that in [37]. However, in [37], $\sigma$ is required no less than the estimation error of the unknown function. Whereas, in practice, it is difficult to determine such an estimation error. In this paper, $\sigma$ is relaxed to be an arbitrary positive number.

Substituting (45) into (25), the closed-loop dynamic equation can be rewritten as

$$
\begin{aligned}
\bar{M}(q) \dot{S}= & -\bar{V}(q, \dot{q}) S-\widehat{f}(x \mid \widehat{\theta})-K_{1} S-K_{2} \operatorname{sig}(S)^{\rho} \\
& -u_{r}+f(x)
\end{aligned}
$$


Multiplying $S^{T}$ to (47) and after some manipulations, we can get

$$
\begin{aligned}
S^{T} \bar{M}(q) \dot{S}= & -S^{T} \bar{V}(q, \dot{q}) S-S^{T} K_{1} S-S^{T} K_{2} \operatorname{sig}(S)^{\rho} \\
& -S^{T} u_{r}+\sum_{i=1}^{2} s_{i} \omega_{f i}(x)+\sum_{i=1}^{2} s_{i} \widetilde{\theta}_{f i}^{T} \xi_{f i}(x) .
\end{aligned}
$$

We use the following adaptation laws to adjust the unknown parameters $\widehat{\theta}_{f i}$ and $\widehat{\varepsilon}_{f}$ :

$$
\begin{aligned}
& \dot{\hat{\theta}}_{f i}=-\mu_{f i} s_{i} \xi_{f i}(x), \\
& \dot{\widehat{\mathcal{\varepsilon}}}_{f}=\eta\|s\|,
\end{aligned}
$$

where $\mu_{f i}>0, i=1,2$, and $\eta>0$.

The properties of the proposed adaptive fuzzy ITSMC law is summarized by the following theorem.

Theorem 11. For the dynamic model (2) of the NWMR with an unknown function $f(x)(26)$, if the integral terminal sliding mode is chosen as (22), the actuator voltage control input with dynamic robust controller $u_{r}(46)$ is designed as (45), and the adaptation laws are (49); then

(1) all the signals in the closed system are bounded;

(2) the sliding variable $S$ will converge to the neighborhood of the integral terminal sliding mode $S=0$ as $\|S\| \leq \delta=$ $\min \left\{\delta_{1}, \delta_{2}\right\}$ in finite time, where

$$
\begin{aligned}
& \delta_{1}=\frac{\left\|\hat{f}\left(x \mid \theta_{f}^{*}\right)-\hat{f}\left(x \mid \hat{\theta}_{f}\right)\right\|+\left\|\widetilde{\varepsilon}_{f}\right\|}{\lambda_{1}}, \\
& \delta_{2}=\left[\frac{\left\|\hat{f}\left(x \mid \theta_{f}^{*}\right)-\widehat{f}\left(x \mid \hat{\theta}_{f}\right)\right\|+\left\|\widetilde{\varepsilon}_{f}\right\|}{\lambda_{2}}\right]^{1 / \rho} .
\end{aligned}
$$

Moreover, the auxiliary velocity tracking error $e_{v i}$ will converge to the region $\left|e_{v i}\right| \leq 2 \delta+\left|e_{v i}(0)\right|, i=1,2$ in finite time.

Proof. Consider the following Lyapunov function candidate:

$$
V_{3}=V_{31}+V_{32} \text {, }
$$

where

$$
\begin{aligned}
V_{31} & =\frac{1}{2} S^{T} \bar{M}(q) S, \\
V_{32} & =\frac{1}{2}\left(\sum_{i=1}^{2} \frac{1}{\mu_{f i}} \widetilde{\theta}_{f i}^{T} \widetilde{\theta}_{f i}+\frac{1}{\eta} \widetilde{\varepsilon}_{f}^{2}\right) .
\end{aligned}
$$

(1) Differentiating $V_{31}$ with respect to time, using (48) and Property 3, we have

$$
\begin{aligned}
\dot{V}_{31}= & -S^{T} K_{1} S-S^{T} K_{2} \operatorname{sig}(S)^{\rho}-S^{T} u_{r}+\sum_{i=1}^{2} s_{i} \omega_{f i}(x) \\
& +\sum_{i=1}^{2} s_{i} \widetilde{\theta}_{f i}^{T} \xi_{f i}(x) .
\end{aligned}
$$

Note that

$$
\sum_{i=1}^{2} s_{i} \omega_{f i}(x) \leq\|S\| \sum_{i=1}^{2} \bar{\omega}_{i} .
$$

Substituting (46) and (54) into (53), the following inequality holds:

$$
\begin{aligned}
\dot{V}_{31} \leq & -S^{T} K_{1} S-S^{T} K_{2} \operatorname{sig}(S)^{\rho}-\|S\|\left(\widehat{\varepsilon}_{f}+\sigma\right) \\
& +\|S\| \sum_{i=1}^{2} \bar{\omega}_{i}+\sum_{i=1}^{2} s_{i} \widetilde{\theta}_{f i}^{T} \xi_{f i}(x) \\
= & -S^{T} K_{1} S-S^{T} K_{2} \operatorname{sig}(S)^{\rho}-\|S\| \sigma-\|S\| \widetilde{\varepsilon}_{f} \\
& +\sum_{i=1}^{2} s_{i} \widetilde{\theta}_{f i}^{T} \xi_{f i}(x) .
\end{aligned}
$$

There results

$$
\begin{aligned}
\dot{V}_{31} \leq & -S^{T} K_{1} S-S^{T} K_{2} \operatorname{sig}(S)^{\rho}-\|S\| \widetilde{\varepsilon}_{f} \\
& +\sum_{i=1}^{2} s_{i} \widetilde{\theta}_{f i}^{T} \xi_{f i}(x) .
\end{aligned}
$$

Differentiating $V_{32}$ with respect to time yields

$$
\dot{V}_{32}=\sum_{i=1}^{2} \frac{1}{\mu_{f i}} \widetilde{\theta}_{f i}^{T} \dot{\tilde{\theta}}_{f i}+\frac{1}{\eta} \widetilde{\varepsilon}_{f} \dot{\vec{\varepsilon}}_{f} .
$$

Combining (56) with (57), we can get

$$
\begin{aligned}
\dot{V}_{31}+\dot{V}_{32} \leq & -S^{T} K_{1} S-S^{T} K_{2} \operatorname{sig}(S)^{\rho} \\
& +\sum_{i=1}^{2} \widetilde{\theta}_{f i}^{T}\left(s_{i} \xi_{f i}(x)+\frac{1}{\mu_{f i}} \dot{\tilde{\theta}}_{f i}\right) \\
& +\widetilde{\varepsilon}_{f}\left(\frac{1}{\eta} \dot{\tilde{\varepsilon}}_{f}-\|S\|\right) .
\end{aligned}
$$

That is,

$$
\begin{aligned}
\dot{V}_{31}+\dot{V}_{32} \leq & -S^{T} K_{1} S-S^{T} K_{2} \operatorname{sig}(S)^{\rho} \\
& +\sum_{i=1}^{2} \widetilde{\theta}_{f i}^{T}\left(s_{i} \xi_{f i}(x)-\frac{1}{\mu_{f i}} \dot{\widehat{\theta}}_{f i}\right) \\
& +\widetilde{\varepsilon}_{f}\left(\frac{1}{\eta} \dot{\widehat{\varepsilon}}_{f}-\|S\|\right) .
\end{aligned}
$$

Applying the adaptation laws (49) into (59), one has

$$
\dot{V}_{31}+\dot{V}_{32} \leq-S^{T} K_{1} S-S^{T} K_{2} \operatorname{sig}(S)^{\rho} .
$$

Clearly, $\dot{V}_{3}=\dot{V}_{31}+\dot{V}_{32} \leq 0$, it is concluded that all the signals $s_{i}, e_{v i}, \widehat{\theta}_{f i}$, and $\widehat{\varepsilon}_{f}$ are bounded. 
(2) According to (56), one gets

$$
\begin{aligned}
\dot{V}_{31} \leq & -S^{T} K_{1} S-S^{T} K_{2} \operatorname{sig}(S)^{\rho}-\|S\| \widetilde{\varepsilon}_{f} \\
& +S^{T}\left[\hat{f}\left(x \mid \theta_{f}^{*}\right)-\widehat{f}\left(x \mid \hat{\theta}_{f}\right)\right] \\
= & -S^{T} K_{1} S-S^{T} K_{2} \operatorname{sig}(S)^{\rho} \\
& +S^{T}\left[\hat{f}\left(x \mid \theta_{f}^{*}\right)-\hat{f}\left(x \mid \hat{\theta}_{f}\right)-\frac{\widetilde{\varepsilon}_{f}}{\|S\|} S\right],
\end{aligned}
$$

which can be further changed into the following two forms:

$$
\begin{gathered}
\dot{V}_{31}<-S^{T}\left\{K_{1}-\operatorname{diag}\left[\widehat{f}\left(x \mid \theta_{f}^{*}\right)-\widehat{f}\left(x \mid \widehat{\theta}_{f}\right)\right.\right. \\
\left.\left.-\frac{\widetilde{\varepsilon}_{f}}{\|S\|} S\right] \operatorname{diag}^{-1}(S)\right\} S-S^{T} K_{2} \operatorname{sig}(S)^{\rho}, \\
\dot{V}_{31}<-S^{T} K_{1} S-S^{T}\left\{K_{2}-\operatorname{diag}\left[\widehat{f}\left(x \mid \theta_{f}^{*}\right)\right.\right. \\
\left.\left.-\widehat{f}\left(x \mid \widehat{\theta}_{f}\right)-\frac{\widetilde{\varepsilon}_{f}}{\|S\|} S\right] \operatorname{diag}^{-1}\left[\operatorname{sig}(S)^{\rho}\right]\right\} \\
\cdot \operatorname{sig}(S)^{\rho} .
\end{gathered}
$$

Denote

$$
\widehat{f}\left(x \mid \theta_{f}^{*}\right)-\widehat{f}\left(x \mid \widehat{\theta}_{f}\right)-\frac{\widetilde{\varepsilon}_{f}}{\|S\|} S=\left[\tilde{f}_{1}, \tilde{f}_{2}\right]^{T} .
$$

For (62), if $\lambda_{1}>\left|\tilde{f}_{i}\right| /\left|s_{i}\right|, i=1,2$, which means the matrix $K_{1}-\operatorname{diag}\left[\widehat{f}\left(x \mid \theta_{f}^{*}\right)-\widehat{f}\left(x \mid \hat{\theta}_{f}\right)-\left(\widetilde{\varepsilon}_{f} /\|S\|\right) S\right] \operatorname{diag}^{-1}(S)$ is positive definite, the similar structure as (35) is kept. Hence, finite time stability is guaranteed. Otherwise, $\left|s_{i}\right| \leq$ $\left|\tilde{f}_{i}\right| /\left|\lambda_{1}\right|, i=1,2$. We can conclude that

$$
\begin{aligned}
\|S\|^{2} & =s_{1}^{2}+s_{2}^{2} \leq \frac{\widetilde{f}_{1}^{2}+\widetilde{f}_{2}^{2}}{\lambda_{1}^{2}} \\
& =\frac{\left\|\widehat{f}\left(x \mid \theta_{f}^{*}\right)-\widehat{f}\left(x \mid \widehat{\theta}_{f}\right)-\left(\widetilde{\varepsilon}_{f} /\|S\|\right) S\right\|^{2}}{\lambda_{1}^{2}} \\
& \leq \frac{\left(\left\|\hat{f}\left(x \mid \theta_{f}^{*}\right)-\widehat{f}\left(x \mid \widehat{\theta}_{f}\right)\right\|+\left\|\widetilde{\varepsilon}_{f}\right\|\right)^{2}}{\lambda_{1}^{2}} ;
\end{aligned}
$$

that is,

$$
\|S\| \leq \frac{\left\|\hat{f}\left(x \mid \theta_{f}^{*}\right)-\hat{f}\left(x \mid \hat{\theta}_{f}\right)\right\|+\left\|\widetilde{\varepsilon}_{f}\right\|}{\lambda_{1}}=\delta_{1} .
$$

Therefore, the region $\|S\| \leq \delta_{1}$ can be reached in finite time.

For (63), if $\lambda_{2}>\left|\tilde{f}_{i}\right| /\left|s_{i}\right|^{\rho}, i=1,2$, which means the matrix $K_{2}-\operatorname{diag}\left[\widehat{f}\left(x \mid \theta_{f}^{*}\right)-\widehat{f}\left(x \mid \widehat{\theta}_{f}\right)-\left(\widetilde{\varepsilon}_{f} /\|S\|\right) S\right] \operatorname{diag}^{-1}\left(S^{\rho}\right)$ is positive definite, the similar structure as (35) is kept.
Hence, finite time stability is guaranteed. Otherwise, $\left|s_{i}\right|^{\rho} \leq$ $\left|\widetilde{f}_{i}\right| /\left|\lambda_{2}\right|, i=1,2$. We can conclude from Lemma 7 that

$$
\begin{aligned}
\|S\|^{4 \rho} & =\left(s_{1}^{2}+s_{2}^{2}\right)^{2 \rho} \leq\left(s_{1}^{2 \rho}+s_{2}^{2 \rho}\right)^{2} \leq\left(\frac{\widetilde{f}_{1}^{2}+\widetilde{f}_{2}^{2}}{\lambda_{2}^{2}}\right)^{2} \\
& =\frac{\left\|\hat{f}\left(x \mid \theta_{f}^{*}\right)-\widehat{f}\left(x \mid \widehat{\theta}_{f}\right)-\left(\widetilde{\varepsilon}_{f} /\|S\|\right) S\right\|^{4}}{\lambda_{2}^{4}} \\
& \leq \frac{\left(\left\|\hat{f}\left(x \mid \theta_{f}^{*}\right)-\widehat{f}\left(x \mid \hat{\theta}_{f}\right)\right\|+\left\|\widetilde{\varepsilon}_{f}\right\|\right)^{4}}{\lambda_{2}^{4}}
\end{aligned}
$$

that is,

$$
\|S\| \leq\left[\frac{\left\|\hat{f}\left(x \mid \theta_{f}^{*}\right)-\widehat{f}\left(x \mid \hat{\theta}_{f}\right)\right\|+\left\|\widetilde{\varepsilon}_{f}\right\|}{\lambda_{2}}\right]^{1 / \rho}=\delta_{2} .
$$

Therefore, the region $\|S\| \leq \delta_{2}$ can be reached in finite time.

By virtue of (66) and (68), the region $\|S\| \leq \delta=$ $\min \left\{\delta_{1}, \delta_{2}\right\}$ can be reached in finite time.

When $\|S\| \leq \delta$, for $i=1,2,\left|s_{i}\right| \leq \delta$. The integral terminal sliding mode (22) can be changed into the following form:

$$
s_{i}=e_{v i}-e_{v i}(0)+\beta_{i} \int_{0}^{t}\left|e_{v i}\right|^{\gamma_{i}} \operatorname{sign}\left(e_{v i}\right) d \tau=\phi_{i},
$$

$$
\left|\phi_{i}\right| \leq \delta \text {, }
$$

or the equivalent form

$$
\begin{aligned}
& e_{v i}-e_{v i}(0) \\
& +\left[\beta_{i}-\frac{\phi_{i}}{\int_{0}^{t}\left|e_{v i}\right|^{\gamma_{i}} \operatorname{sign}\left(e_{v i}\right) d \tau}\right] \int_{0}^{t}\left|e_{v i}\right|^{\gamma_{i}} \operatorname{sign}\left(e_{v i}\right) d \tau \\
& =0 .
\end{aligned}
$$

If $\beta_{i}>\left|\phi_{i}\right| /\left.\left|\int_{0}^{t}\right| e_{v i}\right|^{\gamma_{i}} \operatorname{sign}\left(e_{v i}\right) d \tau \mid,(70)$ is kept in the form of the integral terminal sliding mode. Hence, finite time convergence is guaranteed. Otherwise, $\left.\left|\int_{0}^{t}\right| e_{v i}\right|^{\gamma_{i}} \operatorname{sign}\left(e_{v i}\right) d \tau \mid \leq$ $\left|\phi_{i}\right| / \beta_{i} \leq \delta / \beta_{i}$; the region

$$
\begin{aligned}
\left|e_{v i}\right| & \leq\left.\beta_{i}\left|\int_{0}^{t}\right| e_{v i}\right|^{\gamma_{i}} \operatorname{sign}\left(e_{v i}\right) d \tau|+\delta+| e_{v i}(0) \mid \\
& \leq 2 \delta+\left|e_{v i}(0)\right|
\end{aligned}
$$

can be reached in finite time.

Remark 12. Both the control law (27) and the adaptive fuzzy control law (45) contain a nonlinear term $K_{1} S+K_{2} \operatorname{sig}(S)^{\rho}$ with the form of fast terminal sliding mode, which assures the boundedness of the signals in the closed system and the finite time convergence of the auxiliary velocity tracking error.

Remark 13. According to (66) and (68), the parameters $\lambda_{1}$ and $\lambda_{2}$ can be chosen large enough to make the boundary $\delta$ small. However, increasing the parameters $\lambda_{1}$ and $\lambda_{2}$ will increase the level of control input and will cause implementation problem. 
Remark 14 . The relationship between the auxiliary velocity tracking error $e_{v i}$ and the width of the boundary layer $\delta$ surrounding the integral terminal sliding mode surface $S=0$ is given by (69) and (71).

\section{Simulation Results}

In this section, a simulation will be provided to show the effectiveness of the proposed control strategy.

Referring to [18], the parameters of the NWMR and its actuators are chosen as $m=10 \mathrm{~kg}, I=5 \mathrm{~kg} \cdot \mathrm{m}^{2}, R=0.12 \mathrm{~m}$, $r=0.067 \mathrm{~m}, d=0.3 \mathrm{~m}, N=50, K_{b}=0.026, K_{T}=$ $0.026 \mathrm{Nm} / \mathrm{A}$, and $R_{a}=3.5 \Omega$. The surface friction and the external disturbance are generated by $\bar{F}(\vartheta)+\bar{\tau}_{d}=[5 \sin (2 t)$, $5 \sin (2 t)]^{T}$. In this simulation, the initial posture and velocity of the practical NWMR are taken as $q(0)=[0.1,0.2,0]^{T}$ and $\vartheta=[\nu, \omega]^{T}=[0,0]^{T}$, respectively.

The reference linear velocity and angular velocity are defined as $\nu_{r}(t)=1$ and $\omega_{r}(t)=1$. The trajectory of the reference NWMR is defined as

$$
\begin{aligned}
& \dot{x}_{r}=v_{r} \cos \theta_{r}-\omega_{r} d \sin \theta_{r}, \\
& \dot{y}_{r}=v_{r} \sin \theta_{r}+\omega_{r} d \cos \theta_{r}, \\
& \dot{\theta}_{r}=\omega_{r} .
\end{aligned}
$$

The initial posture of the reference NWMR is taken as $q_{r}(0)=$ $[0,0,0]^{T}$.

The objective of the trajectory tracking control is to design a strategy such that $q(t)$ converges asymptotically to $q_{r}(t)$, while all signals in the derived closed-loop system are able to remain bounded. In the proposed control strategy, an auxiliary velocity controller $\vartheta_{c}$ is designed for the kinematic model to meet the control objective. Then, the actuator voltage control input $u$ is designed for the dynamic model such that $\vartheta$ converges to $\vartheta_{c}$ which is designed at the first step in finite time.

In the actuator voltage control input $u$, the nonlinear function

$$
\begin{aligned}
f(x)= & {\left[f_{1}(x), f_{2}(x)\right]^{T} } \\
= & \bar{M}(q) \dot{\vartheta}_{c}+\bar{M}(q) \Lambda \operatorname{sig}\left(e_{\vartheta}\right)^{\gamma}+\bar{V}(q, \dot{q}) \vartheta_{c} \\
& -\bar{V}(q, \dot{q}) e_{\vartheta}(0)+\bar{V}(q, \dot{q}) \Lambda \int_{0}^{t} \operatorname{sig}\left(e_{\vartheta}\right)^{\gamma} d \tau \\
& +\bar{F}(\vartheta)+\bar{\tau}_{d}
\end{aligned}
$$

is contained, where $x=\left[\vartheta_{c}^{T}, \dot{\vartheta}_{c}^{T}, \vartheta^{T}\right]^{T}$.

We suppose that there is no prior information of the robot parameters such as mass, moment of inertial, friction coefficients, and the external disturbance; that is, the nonlinear function $f_{i}(x), i=1,2$, is assumed to be completely unknown. Two fuzzy systems in the form of (9) are used to approximate $f_{1}(x)$ and $f_{2}(x)$. The fuzzy systems have $x_{1}=v_{c}$, $x_{2}=\omega_{c}, x_{3}=\dot{v}_{c}, x_{4}=\dot{\omega}_{c}, x_{5}=v$, and $x_{6}=\omega$ as inputs;

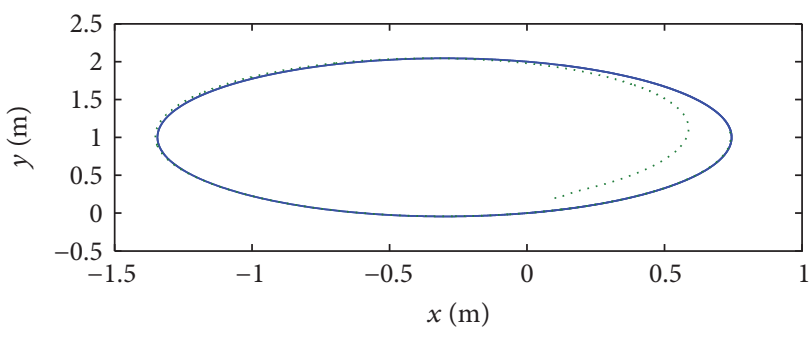

Figure 2: Reference trajectory (-) and actual trajectory $(\cdots)$.

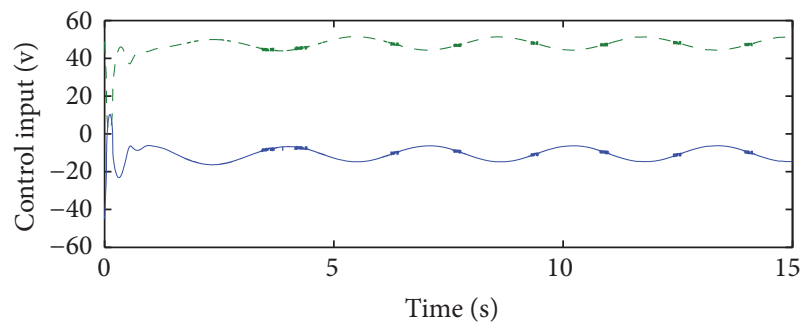

Figure 3: Actuator voltages $u_{1}(-)$ and $u_{2}(--)$.

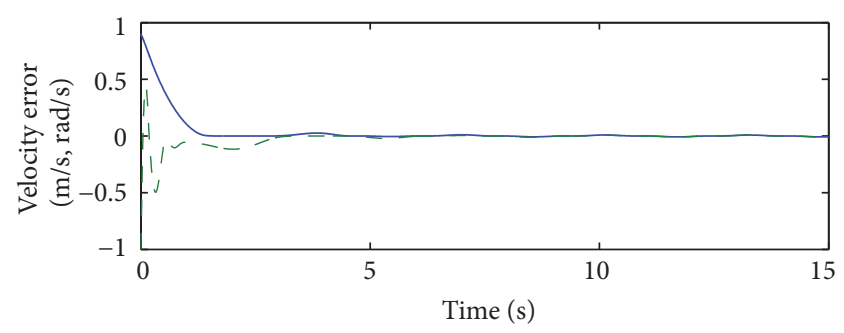

FIGURE 4: Auxiliary velocity tracking errors $e_{91}(-)$ and $e_{92}(--)$.

the fuzzy membership functions for each variable $x_{i}, i=$ $1,2, \ldots, 6$, are chosen as

$$
\begin{aligned}
& \mu_{F_{i}^{1}}\left(x_{i}\right)=\exp \left[-\frac{1}{2}\left(\frac{x_{i}+1.25}{0.6}\right)^{2}\right], \\
& \mu_{F_{i}^{2}}\left(x_{i}\right)=\exp \left[-\frac{1}{2}\left(\frac{x_{i}}{0.6}\right)^{2}\right], \\
& \mu_{F_{i}^{3}}\left(x_{i}\right)=\exp \left[-\frac{1}{2}\left(\frac{x_{i}-1.25}{0.6}\right)^{2}\right] .
\end{aligned}
$$

The initial values of the estimated parameters $\widehat{\theta}_{f i}(0), i=1,2$, and $\widehat{\varepsilon}_{f}(0)$ are all set to 0.01 .

Referring to $[1,31,36]$, the parameters of the control law are chosen as $k_{1}=1, k_{2}=20, k_{3}=10, \beta_{1}=\beta_{2}=1, \gamma_{1}=$ $\gamma_{2}=0.5, k_{11}=k_{12}=k_{21}=k_{22}=20, \rho=0.3, \mu_{f 1}=\mu_{f 2}=$ $0.5, \eta=0.001$, and $\sigma=0.1$.

Using our control strategy to control the NWMR, the simulation results are shown in Figures 2-5. Figure 2 is the trajectory tracking process in $X-Y$ plane of the NWMR, Figure 3 is the actuator voltage control input, Figure 4 is the auxiliary velocity tracking errors, and Figure 5 is the tracking position errors, respectively. From Figure 4, it can be observed that the auxiliary velocity tracking error $e_{91}$ 


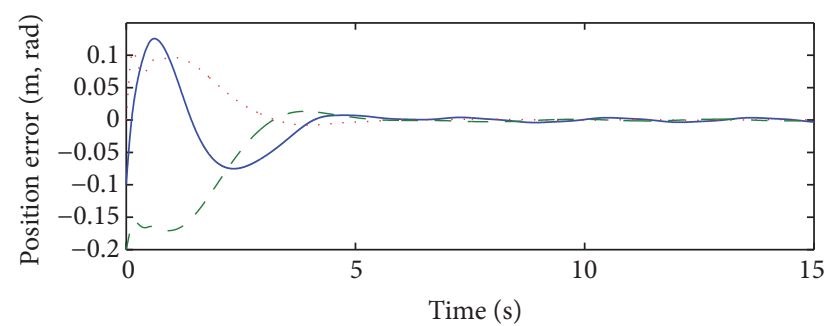

FIGURE 5: Tracking position errors $e_{1}(-), e_{2}(-)$, and $e_{3}(\cdots)$.

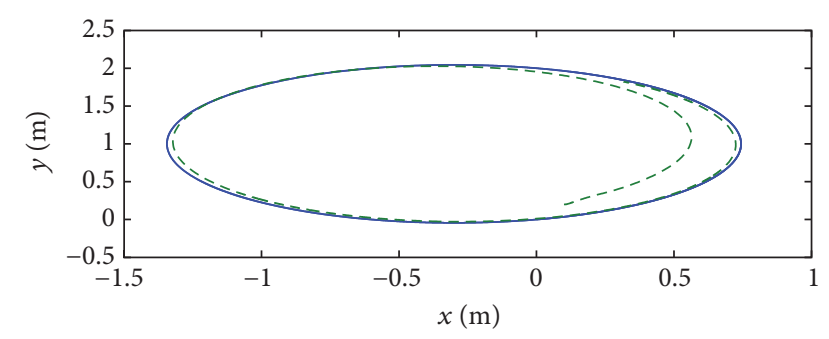

FIGURE 6: Reference trajectory (-) and actual trajectory (- -).

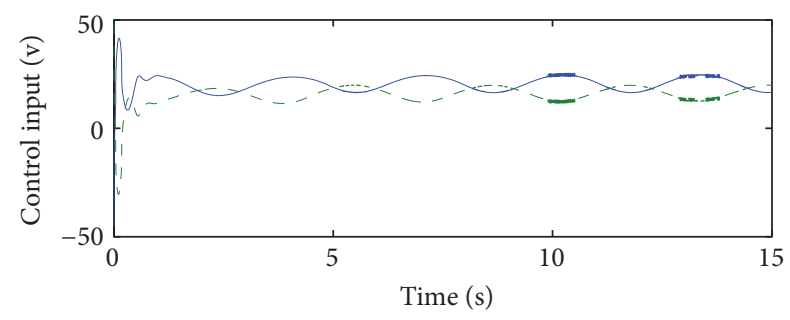

FIGURE 7: Actuator voltages $u_{1}(-)$ and $u_{2}(--)$.

converges to $\left|e_{91}\right| \leq 5 \times 10^{-3}$ in finite time $t=1.389 \mathrm{~s}$ and the auxiliary velocity tracking error $e_{92}$ converges to $\left|e_{92}\right| \leq$ $7 \times 10^{-3}$ in finite time $t=3.052 \mathrm{~s}$, respectively.

In order to compare the proposed integral terminal sliding mode with the integral sliding mode adopted in [25], we use integral sliding mode instead of the integral terminal sliding mode in our control strategy while other design parameters are the same as the corresponding design parameters used in the above simulation. The corresponding simulation results are shown in Figures 6-9. From Figure 8, it can be observed that the auxiliary velocity tracking error $e_{91}$ converges to $\left|e_{91}\right| \leq 3 \times 10^{-2}$ in finite time $t=13.47 \mathrm{~s}$ and the auxiliary velocity tracking error $e_{92}$ converges to $\left|e_{{ }_{9}}\right| \leq$ $4 \times 10^{-2}$ in finite time $t=14.05 \mathrm{~s}$, respectively.

It is observed that the actual velocity can track the auxiliary velocity in less time using the control strategy proposed in this paper from Figures 4 and 8. As a result, the practical NWMR can track the reference NWMR asymptotically with faster response from Figures 2 and 6. Meanwhile, this favorable performance was obtained with no prior information of the robot parameters such as mass, moment of inertial, friction coefficients, and the external disturbance.

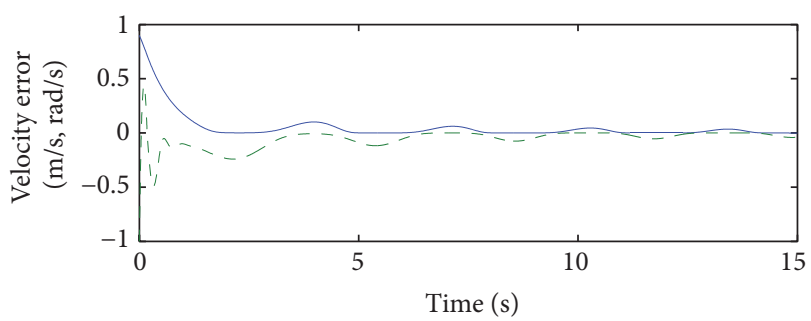

FIGURE 8: Auxiliary velocity tracking errors $e_{91}(-)$ and $e_{92}(--)$.

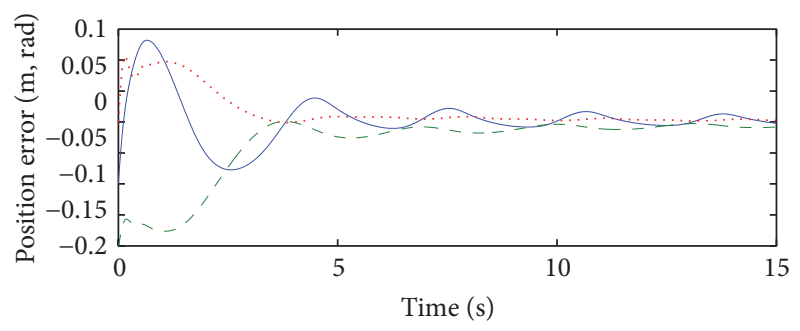

FIGURE 9: Tracking position errors $e_{1}(-), e_{2}(-)$, and $e_{3}(\cdots)$.

\section{Conclusions}

In this paper, a control strategy has been proposed for the trajectory tracking problem of the NWMR with parameter uncertainties and external disturbances. In this study, we take the wheel actuator dynamics into system dynamics and choose the actuator voltage as the control input. The FLS is adopted to estimate the unknown function coming from parameter uncertainties and external disturbances. An adaptive fuzzy integral terminal sliding mode controller is integrated with an auxiliary velocity controller. It has been shown that all the signals in the closed system are bounded and the auxiliary velocity tracking errors converge to a small neighborhood of the origin in finite time. Hence, the tracking position errors converge asymptotically to zeros with faster response than other existing controllers. Simulation results have been provided to show the feasibility of the proposed control strategy. However, wheel skidding and slipping are unavoidable due to tire deformation and other reasons in real environments. In the future, we will extend our results to the trajectory tracking control of the WMR with wheel skidding and slipping.

\section{Conflicts of Interest}

The authors declare that they have no conflicts of interest.

\section{Acknowledgments}

This work was supported by the Natural Science Foundation of Tianjin, China, under Grants 15JCYBJC47800.

\section{References}

[1] Y. Kanayama, Y. Kimura, F. Miyazaki, and T. Noguchi, "A stable tracking control method for an autonomous mobile robot," in 
Proceedings of the IEEE International Conference on Robotics and Automation, vol. 1, pp. 384-389, May 1990.

[2] T. H. Bui, T. T. Nguyen, T. L. Chung, and S. B. Kim, "A simple nonlinear control of a two-wheeled welding mobile robot," International Journal of Control, Automation and Systems, vol. 1, no. 1, pp. 35-42, 2003.

[3] R. Fierro and F. L. Lewis, "Control of a nonholonomic mobile robot: backstepping kinematics into dynamics," in Proceedings of the 34th IEEE Conference on Decision and Control, pp. 38053810, December 1995.

[4] T. Appala and A. Ghosal, "Tip over stability analysis of a threewheeled mobile robot capable of traversing uneven terrains without slip," Applied Mechanics and Materials, vol. 110-116, pp. 2940-2947, 2012.

[5] T. Appala and A. Ghosal, "A mobile robot with a two-degree-offreedom suspension for traversing uneven terrain with minimal slip: modeling, simulation and experiments," Mechanism and Machine Theory, vol. 93, pp. 83-97, 2015.

[6] D. Wang and C. B. Low, "Modeling and analysis of skidding and slipping in wheeled mobile robots: control design perspective," IEEE Transactions on Robotics, vol. 24, no. 3, pp. 676-687, 2008.

[7] T. Fukao, H. Nakagawa, and N. Adachi, "Adaptive tracking control of a nonholonomic mobile robot," IEEE Transactions on Robotics and Automation, vol. 16, no. 5, pp. 609-615, 2000.

[8] B. S. Park, S. J. Yoo, J. B. Park, and Y. H. Choi, "Adaptive output-feedback control for trajectory tracking of electrically driven non-holonomic mobile robots," IET Control Theory and Applications, vol. 5, no. 6, pp. 830-838, 2011.

[9] J. Huang, C. Wen, W. Wang, and Z.-P. Jiang, "Adaptive output feedback tracking control of a nonholonomic mobile robot," Automatica, vol. 50, no. 3, pp. 821-831, 2014.

[10] S. J. Yoo, "Approximation-based adaptive control for a class of mobile robots with unknown skidding and slipping," International Journal of Control, Automation and Systems, vol. 10, no. 4, pp. 703-710, 2012.

[11] Y. Koubaa, M. Boukattaya, and T. Dammak, "An adaptive control for uncertain mobile robot considering skidding and slipping effects," in Proceedings of the 5th International Conference on Systems and Control (ICSC '16), pp. 13-19, May 2016.

[12] J. Wu, G. Xu, and Z. Yin, "Robust adaptive control for a nonholonomic mobile robot with unknown parameters," Journal of Control Theory and Applications, vol. 7, no. 2, pp. 212-218, 2009.

[13] L. Xin, Q. Wang, J. She, and Y. Li, "Robust adaptive tracking control of wheeled mobile robot," Robotics and Autonomous Systems, vol. 78, pp. 36-48, 2016.

[14] M. Chen, "Disturbance attenuation tracking control for wheeled mobile robots with skidding and slipping," IEEE Transactions on Industrial Electronics, vol. 64, no. 4, pp. 33593368, 2017.

[15] A. Zou, Z. Hou, M. Tan, and Z. Zhao, "Tracking control of a nonholonomic mobile robot using a fuzzy-based approach," in Fuzzy Systems and Knowledge Discovery, vol. 4223 of Lecture Notes in Computer Science, pp. 826-835, Springer, Berlin, Germany, 2006.

[16] T. Das and I. N. Kar, "Design and implementation of an adaptive fuzzy logic-based controller for wheeled mobile robots," IEEE Transactions on Control Systems Technology, vol. 14, no. 3, pp. 501-510, 2006.

[17] Z.-G. Hou, A.-M. Zou, L. Cheng, and M. Tan, "Adaptive control of an electrically driven nonholonomic mobile robot via backstepping and fuzzy approach," IEEE Transactions on Control Systems Technology, vol. 17, no. 4, pp. 803-815, 2009.
[18] T. Q. Vinh, N. H. Giap, T.-W. Kim, M.-G. Jeong, J.-H. Shin, and W.-H. Kim, "Adaptive robust fuzzy control and implementation for path tracking of a mobile robot," in Proceedings of the IEEE International Symposium on Industrial Electronics (IEEE ISIE '09), pp. 1943-1949, July 2009.

[19] R. Fierro and F. L. Lewis, "Control of a nonholonomic mobile robot using neural networks," IEEE Transactions on Neural Networks, vol. 9, no. 4, pp. 589-600, 1998.

[20] W. Zeng, Q. Wang, F. Liu, and Y. Wang, "Learning from adaptive neural network output feedback control of a unicycle-type mobile robot," ISA Transactions, vol. 61, pp. 337-347, 2016.

[21] J.-M. Yang and J.-H. Kim, "Sliding mode control for trajectory tracking of nonholonomic wheeled mobile robots," IEEE Transactions on Robotics and Automation, vol. 15, no. 3, pp. 578-587, 1999.

[22] D. Chwa, "Sliding-mode tracking control of nonholonomic wheeled mobile robots in polar coordinates," IEEE Transactions on Control Systems Technology, vol. 12, no. 4, pp. 637-644, 2004.

[23] J. K. Lee, Y. H. Choi, and J. B. Park, "Sliding mode tracking control of mobile robots with approach angle in cartesian coordinates," International Journal of Control, Automation and Systems, vol. 13, no. 3, pp. 718-724, 2015.

[24] Y. Hu and S. X. Yang, "A fuzzy neural dynamics based tracking controller for a nonholonomic mobile robot," in Proceedings of the IEEE/ASME International Conference on Advanced Intelligent Mechatronics (AIM '03), pp. 205-210, IEEE, July 2003.

[25] M. Yue, S. Wang, and Y. Zhang, "Adaptive fuzzy logic-based sliding mode control for a nonholonomic mobile robot in the presence of dynamic uncertainties," Proceedings of the Institution of Mechanical Engineers, Part C: Journal of Mechanical Engineering Science, vol. 229, no. 11, pp. 1979-1988, 2015.

[26] S. Tong, B. Huo, and Y. Li, “Observer-based adaptive decentralized fuzzy fault-tolerant control of nonlinear large-scale systems with actuator failures," IEEE Transactions on Fuzzy Systems, vol. 22, no. 1, pp. 1-15, 2014.

[27] S. Tong, T. Wang, and Y. Li, "Fuzzy adaptive actuator failure compensation control of uncertain stochastic nonlinear systems with unmodeled dynamics," IEEE Transactions on Fuzzy Systems, vol. 22, no. 3, pp. 563-574, 2014.

[28] Y. Li, S. Tong, and T. Li, “Observer-based adaptive fuzzy tracking control of MIMO stochastic nonlinear systems with unknown control directions and unknown dead zones," IEEE Transactions on Fuzzy Systems, vol. 23, no. 4, pp. 1228-1241, 2015.

[29] Y. Li, S. Tong, and T. Li, "Hybrid fuzzy adaptive output feedback control design for uncertain MIMO nonlinear systems with time-varying delays and input saturation," IEEE Transactions on Fuzzy Systems, vol. 24, no. 4, pp. 841-853, 2016.

[30] S. Tong, S. Sui, and Y. Li, "Fuzzy adaptive output feedback control of MIMO nonlinear systems with partial tracking errors constrained," IEEE Transactions on Fuzzy Systems, vol. 23, no. 4, pp. 729-742, 2015.

[31] W. Shi, "Adaptive fuzzy control for multi-input multi-output nonlinear systems with unknown dead-zone inputs," Applied Soft Computing Journal, vol. 30, pp. 36-47, 2015.

[32] S. T. Venkataraman and S. Gulati, "Control of nonlinear systems using terminal sliding modes," in Proceedings of the American Control Conference, pp. 891-893, June 1992.

[33] M. Zhihong, A. P. Paplinski, and H. R. Wu, "A robust mimo terminal sliding mode control scheme for rigid robotic manipulators," IEEE Transactions on Automatic Control, vol. 39, no. 12, pp. 2464-2469, 1994. 
[34] K.-B. Park and J.-J. Lee, "Comments on 'a robust MIMO terminal sliding mode control scheme for rigid robotic manipulators,' IEEE Transactions on Automatic Control, vol. 41, no. 5, pp. 761-762, 1996.

[35] Y. Feng, X. Yu, and Z. Man, "Non-singular terminal sliding mode control of rigid manipulators," Automatica, vol. 38, no. 12, pp. 2159-2167, 2002.

[36] S. Yu, X. Yu, B. Shirinzadeh, and Z. Man, "Continuous finitetime control for robotic manipulators with terminal sliding mode," Automatica, vol. 41, no. 11, pp. 1957-1964, 2005.

[37] L. Wang, T. Chai, and L. Zhai, "Neural-network-based terminal sliding-mode control of robotic manipulators including actuator dynamics," IEEE Transactions on Industrial Electronics, vol. 56, no. 9, pp. 3296-3304, 2009.

[38] T. Madani, B. Daachi, and K. Djouani, "Non-singular terminal sliding mode controller: application to an actuated exoskeleton," Mechatronics, vol. 33, pp. 136-145, 2016.

[39] V. Nekoukar and A. Erfanian, "Adaptive fuzzy terminal sliding mode control for a class of MIMO uncertain nonlinear systems," Fuzzy Sets and Systems, vol. 179, no. 1, pp. 34-49, 2011.

[40] S. Khoo, L. Xie, and Z. Man, "Integral terminal sliding mode cooperative control of multi-robot networks," in Proceedings of the IEEE/ASME International Conference on Advanced Intelligent Mechatronics (AIM '09), pp. 969-973, IEEE, Singapore, July 2009.

[41] C.-S. Chiu, "Derivative and integral terminal sliding mode control for a class of MIMO nonlinear systems," Automatica, vol. 48, no. 2, pp. 316-326, 2012.

[42] A. Vladimir, R. Zorich, and R. Cooke, Mathmatical Analysis I, Springer, 2008.

[43] D. Huang, J. Zhai, W. Ai, and S. Fei, "Disturbance observerbased robust control for trajectory tracking of wheeled mobile robots," Neurocomputing, vol. 198, pp. 74-79, 2016.

[44] J. Slotine and W. Li, Applied Nonlinear Control, Prentice Hall, Englewood Cliffs, NJ, USA, 1991. 


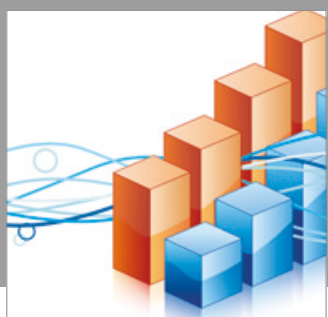

Advances in

Operations Research

vatersals

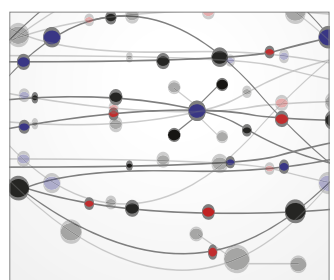

\section{The Scientific} World Journal
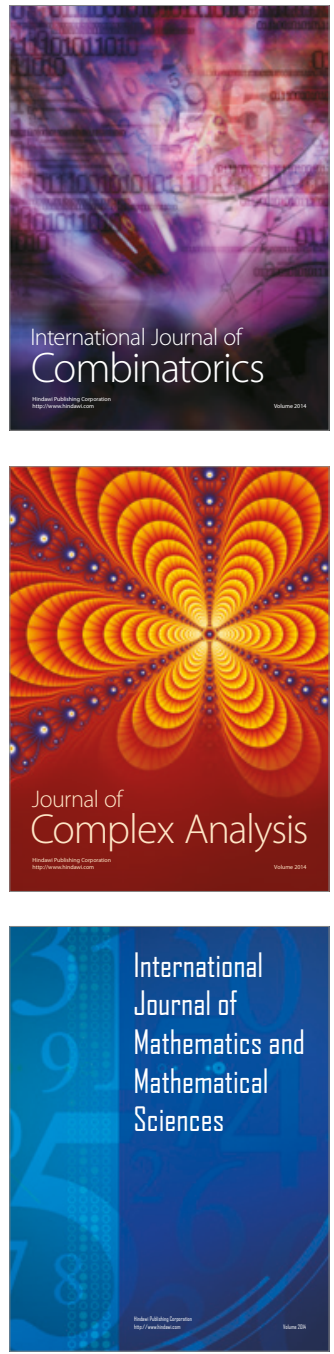
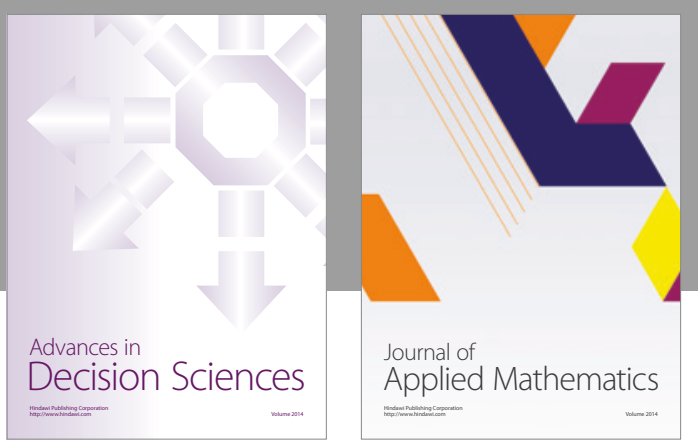

Algebra

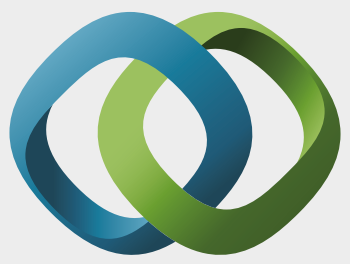

\section{Hindawi}

Submit your manuscripts at

https://www.hindawi.com
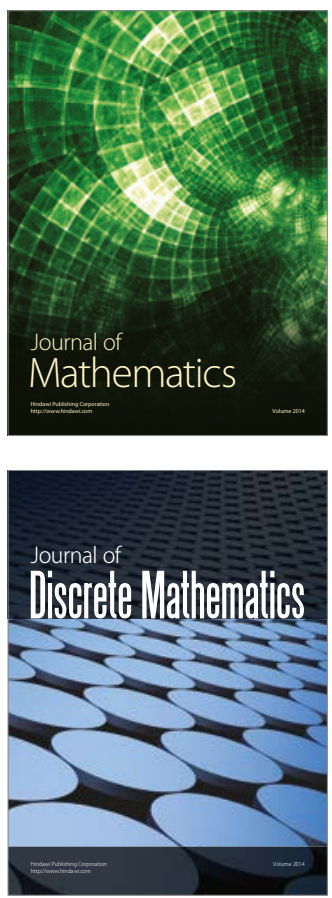

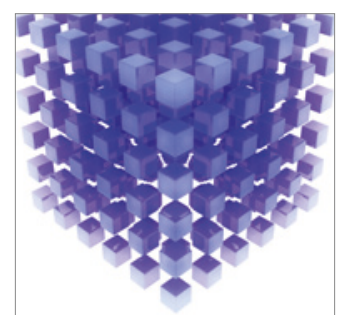

Mathematical Problems in Engineering
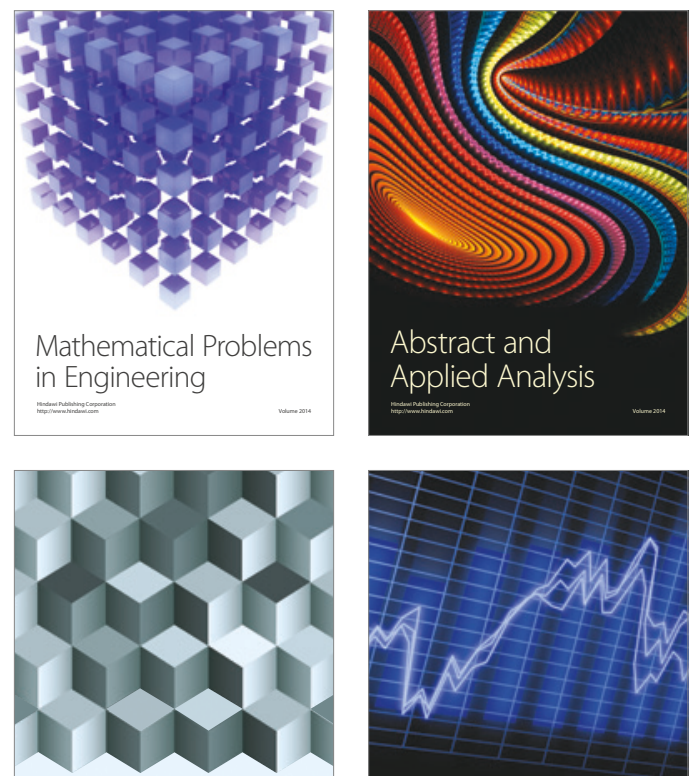

Journal of

Function Spaces

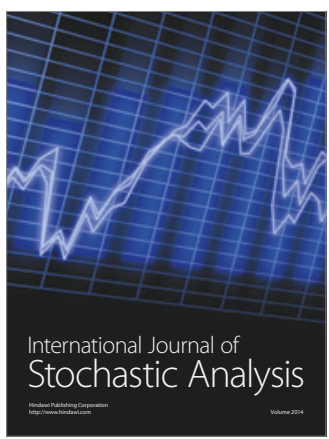

Probability and Statistics
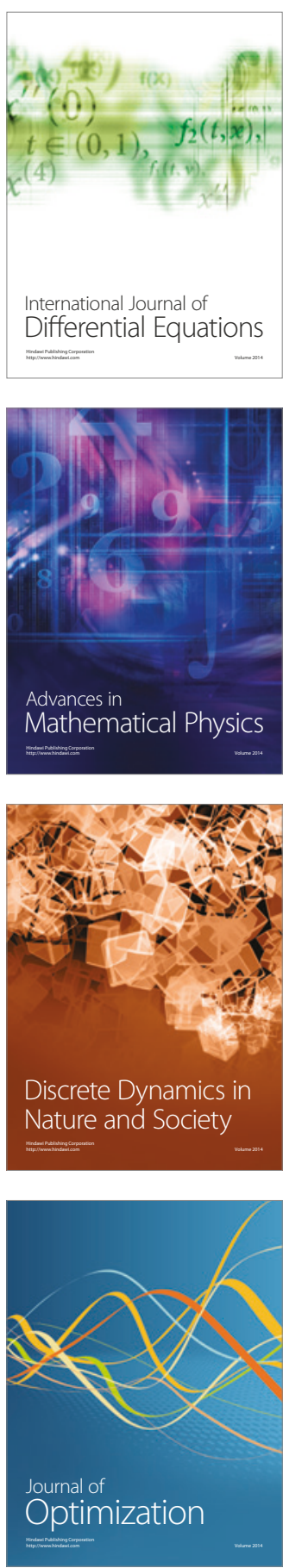